\title{
Surface and bulk contributions in magnetic linear dichroism in the angular dependence from ferromagnetic transition metals
}

\author{
Francesco Bruno, Giancarlo Panaccione, Alberto Verdini, Roberto Gotter, and Luca Floreano \\ INFM, TASC Laboratory, S.S. 14 Km 163.5, I-34012 Trieste, Italy \\ Piero Torelli, Maurizio Sacchi, and Fausto Sirotti \\ Laboratoire pour l'Utilisation du Rayonnement Electromagnetique, CNRS-CEA-MESR, Boîte Postale 34, F-91898 Orsay, France
}

\author{
Alberto Morgante \\ INFM-Dipartimento di Fisica dell' Universita' di Trieste, I-34100 Trieste, Italy \\ and INFM, TASC Laboratory, S.S. 14 Km 163.5, I-34012 Trieste, Italy
}

\author{
Giorgio Rossi \\ INFM-Dipartimento di Fisica dell' Universita' di Modena e Reggio Emilia, I-41100 Modena, Italy \\ and INFM, TASC Laboratory, S.S. 14 Km 163.5, I-34012 Trieste, Italy \\ (Received 9 November 2001; revised manuscript received 7 March 2002; published 10 July 2002)
}

\begin{abstract}
The effects of photoelectron diffraction on the magnetic linear dichroism in the angular distribution (MLDAD) signal have been measured for experiments on crystalline iron and cobalt. Experimental photoemission data of $\mathrm{Fe} 3 p$ and $\mathrm{Co} 3 p$ core levels have been obtained in chiral geometry from bulk $\mathrm{Fe}(100)$ and from $\mathrm{Co} / \mathrm{Fe}(100)$ and $\mathrm{Fe} / \mathrm{Co} / \mathrm{Fe}(100)$ epitaxial interfaces. A prominent forward-scattering peak is observed at normal emission and, correspondingly, a severe reduction of the Fe and Co magnetic dichroism asymmetry. The comparison between full multiple-scattering calculations and experimental results provides a rationale for understanding the role of photoelectron diffraction in MLDAD experiments. In this connection we show that both surface and bulk atoms contribute to the measured dichroic signal, but the line shape of bulk contribution to the spectra can be retrieved.
\end{abstract}

DOI: 10.1103/PhysRevB.66.024417

PACS number(s): 75.70.Rf, 75.25.+z, 71.70.Ej, 79.60.-i

\section{INTRODUCTION}

The magnetic properties of surfaces and interfaces are peculiar, ${ }^{1}$ but their experimental investigation is difficult in most magnetometries, due to the small surface to bulk signal ratio. The surface science approach to magnetism has developed several methods of investigation of magnetic order at surfaces and interfaces, often based on photoemission (PE). Magneto-optic effects in PE from core levels have provided useful information on surface magnetization, spin-wave stiffness of surfaces, and interface coupling, but the quantitative analysis of the data is made difficult by the concurrent signals of bulk, surface and subsurface layers, and by the general phenomenon of photoelectron diffraction (PED) ${ }^{2-7}$ Ten years of light polarization and sample magnetization dependent PE experiments have shown indirectly or directly that PED effects can modify not only the intensity of core level peaks, as a function of angle and kinetic energy, but also their overall magnetic dichroism. For an appropriate choice of the experimental geometry, the PE integrated intensity (not spin selected) may depend on the photon polarization and/or on the sample magnetization, due to the interactions between the core-hole and the electronic states carrying the magnetic moment. In particular, using linearly polarized light in chiral geometry, ${ }^{8}$ one can measure magnetic linear dichroism in the angular distribution (MLDAD) of PE. Since the first observation by Roth et al., ${ }^{8}$ a number of theoretical and experimental efforts have clarified the underlying physics and the possible applications of MLDAD ${ }^{9-15}$ The problem of distinguishing surface and subsurface or bulk contributions in the MLDAD-PE experiments has been addressed according to the specific spectroscopic features of core levels in various materials. Whenever large surface energy shifts can be directly energy resolved in the experiments, as in the case of $4 f$ PE from rare earths, the direct observation of surface and bulk magnetic dichroism has been possible. ${ }^{16}$ However, the case of ferromagnetic transition metals (TM), much relevant for magnetism, does not show energyresolved core-level features in $\mathrm{PE}$, frustrating then the attempts of disentangling the surface contribution from the bulk one.

In order to improve the general understanding of the MLDAD experiments and therefore to better assess the ultimate relevance of this approach in addressing surface magnetism we have performed a set of experiments in various geometries exploiting the flexibility of a multianalyzer apparatus and linearly polarized synchrotron radiation on $\mathrm{Fe} 3 p$ and $\mathrm{Co} 3 p$ signals from $\mathrm{Fe}(100)$ surfaces and $\mathrm{Co} / \mathrm{Fe}(100)$ and $\mathrm{Fe} / \mathrm{Co} / \mathrm{Fe}(100)$ interfaces. The samples were chosen as to maintain the same crystalline structure thanks to epitaxy while varying the surface vs subsurface contribution of both iron and cobalt. The full multiple-scattering calculations for a $3 p$ core photoemission signal in a [100] oriented bcc crystal with Fe lattice parameter, and ignoring spin effects, gives the pattern of PED for surface and subsurface/bulk signals as a function of kinetic energy. As a function of the emission angle, the bulk contribution displays large oscillations with respect to the smooth behavior of the surface contribution. 

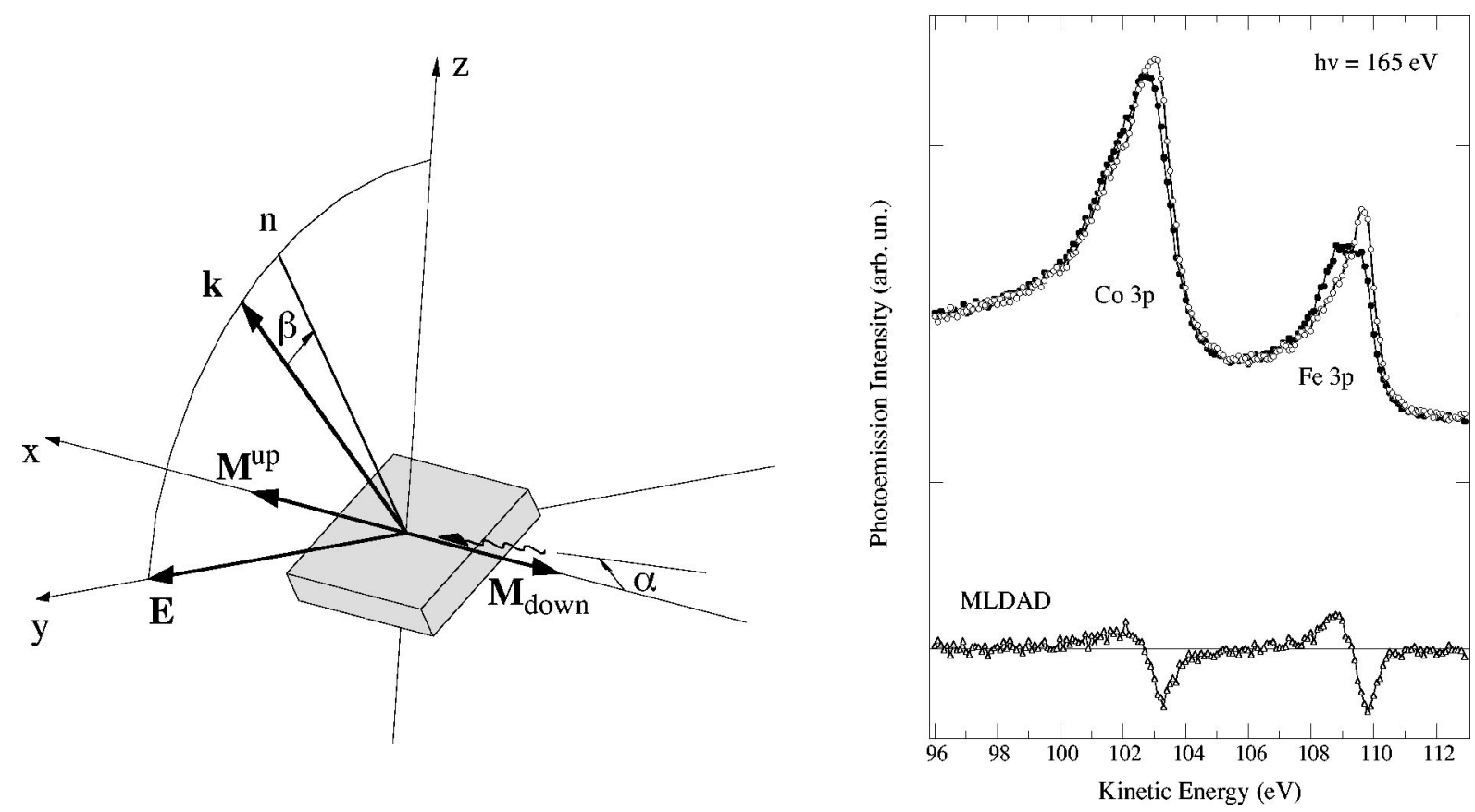

FIG. 1. Left: Chiral geometry of the experiment. The magnetization $\mathbf{M}$ is imposed in a direction perpendicular to the electric field $\mathbf{E}$. The incident Radiation is at angle $\alpha=5^{\circ}$. Angular scans may be performed either by the rotation of the crystal surface around the beam axis (fixed chirality) or by scanning the analyzer position. The measurements presented in this paper were performed varying the polar angle $\beta$ by rotating the surface normal $n$. The photoemission direction was set to $45^{\circ}$ from the photon electric-field direction $\mathbf{E}$. The magnitude of MLDAD is only affected by the degree of chirality imposed by the varying analyzer position. Fe and Co $3 p$ magnetization. Right: Fe and Co $3 p$ magnetization dependent spectra with their relative MLDAD signals.

The systematic analysis of the whole set of experimental data and the comparison to calculations allows one to separate the surface and bulk MLDAD signals. By this procedure we have filtered out the line shape of the bulk contribution, which can be used as a reference PE bulklike signal. With respect to the phenomenological discussion of previous experiments on $\mathrm{Fe}(100)$ we have obtained an independent evidence of the role of PED in enhancing the bulk contribution in particular combinations of geometry and energy. This opens the possibility of addressing at a quantitative level the analysis of PE-MLDAD data for clean TM's surfaces and interfaces.

\section{MLDAD AND PHOTOELECTRON DIFFRACTION}

The MLDAD signal in a $l=1$ core-level PE spectrum is shown for Fe $3 p$ and Co $3 p$ in Fig. 1 as obtained in the chiral geometry shown schematically in the left panel of the same figure. MLDAD can be described by (a) the up-down feature, (b) the magnetic asymmetry $A_{M L D A D}$, i.e., the normalized magnetic signal, usually defined as ( $I^{\text {up }}$ $\left.-I^{\text {down }}\right) /\left(I^{\text {up }}+I^{\text {down }}\right)$ where $I^{\text {up }}, I^{\text {down }}$ are the measured magnetization dependent PE intensities, and (c) the splitting value, i.e., the separation in energy between the maximum and the minimum of the dichroism curve. The first characteristic (a) is related to the alignment of the orbital moment of the core hole with respect to the quantization axis. ${ }^{17}$ The magnetic asymmetry $A_{M L D A D}$ was demonstrated experimentally to be proportional to the magnetic order parameter. ${ }^{9}$ The MLDAD energy splitting in TM has been explained by means of an anomalous Zeeman-like effect due to the effective exchange field of the split $d$ band, acting on the core hole in the photoemission process. The single-electron analysis of the core-hole multiplet was proposed at a very basic level by Rossi et al. ${ }^{18,19}$ Several calculations based on various electronic structure approaches produced similar results, albeit based on more appropriate physics ground. It was also proposed that the splitting energy must bear a proportionality to the exchange field value, i.e., to the spin magnetic moment of the photoemitting atom. ${ }^{9,22}$ Highly reliable MLDAD results have been obtained by performing experiments in a fixed geometry, so as to limit the influence of diffraction effects. ${ }^{10}$ However, other experiments were performed to show the presence of strong PED effects on TM crystal surfaces $^{20-22}$ and have been discussed with the support of full multiple-scattering calculations on representative clusters. ${ }^{21}$ At present, the main experimental evidences may be summarized as follows. (i) The $A_{M L D A D}$ behavior for a TM crystal as a function of both emission angle and electron kinetic energy presents strong deviations with respect to the atomiclike $A_{M L D A D}$ behavior measured in polycrystalline and/or amorphous ferromagnetic films. ${ }^{21,22}$ (ii) The up-down MLDAD feature may undergo a sign reversal (i.e., from updown to down-up) when scanning the take-off angle at particular values of kinetic energy, as shown in Refs. 22 and 24, and 25 for $\mathrm{Fe} 3 p$ and $\mathrm{Fe} 2 p$, Co $2 p$, respectively. (iii) The minimum value of the $A_{M L D A D}$ may not correspond to the maximum of the integrated PE signal, i.e. it is not placed at normal emission. ${ }^{20,21}$ The origin of the sign reversal of MLDAD was tentatively ascribed to PED effects, and the 
possibility of exploiting this fact to separate bulk and surface contributions in the dichroic signal was pointed out. ${ }^{22}$ It is worth noting that most of the MLDAD-PED experiments reported in the literature have been performed using $h \nu$ $=1253 \mathrm{eV}$ or $h \nu=1484 \mathrm{eV}$ from $\mathrm{Mg}$ and $\mathrm{Al} K \alpha$ radiation yielding photoelectron kinetic energies high enough to justify a kinematic analysis that neglects multiple-scattering effects and considers the forward-scattering intensities as the prevailing contribution in PED. These experiments on Fe $2 p$ and $3 p$ have intrinsically a limited surface sensitivity.

Our present experiment has been designed with the aim to study the relationship between MLDAD signal and PED in the surface sensitive regime of PE, i.e., by using linearly polarized monochromatic synchrotron radiation with corelevel kinetic energies lower than $250 \mathrm{eV}$, on samples where alternatively Fe and Co are the only species at the surface or in the subsurface layer.

\section{EXPERIMENT}

MLDAD-PED experiments were performed at the ALOISA beamline in ELETTRA. ${ }^{26}$ Seven hemispherical electron analyzers are hosted inside the experimental chamber. In particular, two of these analyzers are mounted on a rotating frame; this experimental setup, together with the rotation of the whole chamber around the beam axis, allows one to select freely any orientation of the emission direction with respect to the sample surface. The extremely high flexibility in the experimental geometry is particularly suited for polarization dependent PED investigations: ${ }^{27}$ almost any chiral configuration can be easily and accurately selected. The sample was mounted on a six degrees of freedom manipulator, with a $0.01^{\circ}$ accuracy selection for incidence angle on the surface, for surface azimuthal orientation as well as for the surface orientation with respect to the photon electric field (rotation around the beam axis). Details of sample mounting to perform MLDAD experiments can be found in Ref. 9. Figure 1 shows the geometry of the experiment for the MLDAD measurements: PE spectra were measured at room temperature with the magnetization $\mathbf{M}$ imposed parallel to the surface, along the beam direction, and the angle $\alpha$ was set to $5^{\circ}$, where $\alpha$ is defined as the angle measured from the surface plane (Fig. 1). Once a chiral configuration was chosen, angular scans were performed through a rotation of the crystal around the magnetization direction, so as to keep fixed the degree of chirality for all emission angles; magnetization dependent spectra were collected by reversing the magnetization direction applying a short current pulse through the windings of a horse-shoe magnet. The surface normal was set to $45^{\circ}$ from the photon polarization so that PE intensity displays its maximum in the polar range between normal emission (maximum probing depth) and the polar position $\theta=45^{\circ}$ of the photon polarization vector (maximum of atomic $p$ level cross section). On the other hand, the magnitude of MLDAD is only affected by the degree of chirality imposed by the varying analyzer position. Angular acceptance was $\pm 0.5^{\circ}$ and overall energy resolution was $200 \mathrm{meV}$; base pressure was in the $10^{-11}$ mbars range. Complete MLDAD data sets were obtained from polycrys- talline $\mathrm{Fe}$ grown on $\mathrm{Fe}_{30} \mathrm{Ni}_{50} \mathrm{~B}_{20}$, providing reference spectra, free from PED modulations, i.e., modulated only by the atomic photoionization angular matrix elements. ${ }^{28}$ Clean (carbon and sulfur free) $\mathrm{Fe}(100)$ surfaces were obtained by sputtering-annealing cycles $\left(\mathrm{Ar}^{+} 1 \mathrm{keV}, 600^{\circ} \mathrm{C}\right)$, as well as by monitoring the Auger lines of principal contaminants. We observed a degradation in the surface cleanliness after roughly $10 \mathrm{~h}$. A complete angular and/or energy scan could be performed before a new cleaning cycle was required. Several data sets were obtained reproducibly. The epitaxial overgrowths of $\mathrm{Co}$ and $\mathrm{Fe}$ on $\mathrm{Fe}(100)$ surface were obtained in situ, by $e$-beam evaporation in a vacuum of 2 $\times 10^{-10}$ mbars. Co is known to grow epitaxially on $\mathrm{Fe}(100)$ up to 6 monolayers, continuing the bcc lattice structure of the substrate. Likewise a further overgrowth of $\mathrm{Fe}$ on the bcc Co maintains the same structure as that of the substrate with negligible relaxation. ${ }^{31}$ By creating a topmost layer of cobalt one expects two facts: (a) the enhanced magnetic moment of surface $\mathrm{Fe}(100)$ should be reduced by the termination with cobalt; (b) to induce similar PED effects for the iron PE signals, including also the normal emission direction, since each $\mathrm{Fe}$ atom has a similar scattering environment. By a further growth of a new topmost layer of iron on $\mathrm{Co} / \mathrm{Fe}(100)$ one expects that the bulk iron signal is rather severely damped, while the surface contribution, including PED effects are fully active. Conversely, the cobalt signal should display a pure surface PED behavior in the $\mathrm{Co} / \mathrm{Fe}(100)$ sample and a pure subsurface layer behavior in $\mathrm{Fe} / \mathrm{Co} /$ $\mathrm{Fe}(100)$. The analysis of the whole data set does allow one to establish, on the experimental basis, the different patterns of the $\mathrm{Fe}(100) 3 p$ PED signal from surface and subsurface-bulk atoms.

The experimental PED patterns for surface and subsurface-bulk $3 p$ signals in $\mathrm{Fe}(100)$ have been compared to the layer-resolved PED calculation (obtained using the MSCD code by Chen and Van Hove $\left.{ }^{29}\right)$. An hemispherical cluster of 180 atoms with the Fe bcc(001) structure was used. The calculation takes into account the interference between the final state angular momenta $(l=0$ and $l=2)$, which contributes to the photoelectron emission from the $p$ core level $(l=1)$, including multiple-scattering contributions up to the sixth order. Further nonstructural parameters, such as the muffin tin radius and the Debye temperature, were set to the values that proved to be satisfactory in previous PED studies of Fe structure. ${ }^{30}$ The surface and bulk contributions were simply obtained by switching on the emission from the surface (bulk) atoms, treating the bulk (surface) atoms as scatterers only. The total photoelectron intensity (surface+bulk) is the incoherent sum of the surface and bulk simulated intensities.

\section{RESULTS AND DISCUSSION}

According to Refs. 22 and 28 a minimum for the bcc $\mathrm{Fe}(100) 3 p A_{M L D A D}$ vs photon energy is found near $165 \mathrm{eV}$. Moreover, a minimum is found around normal emission as a function of the emission angle, at $h \nu=165 \mathrm{eV}$, to be compared to the smooth behavior measured for the poly-Fe. ${ }^{22,28}$ Figure 2 presents data points for the magnetization averaged 


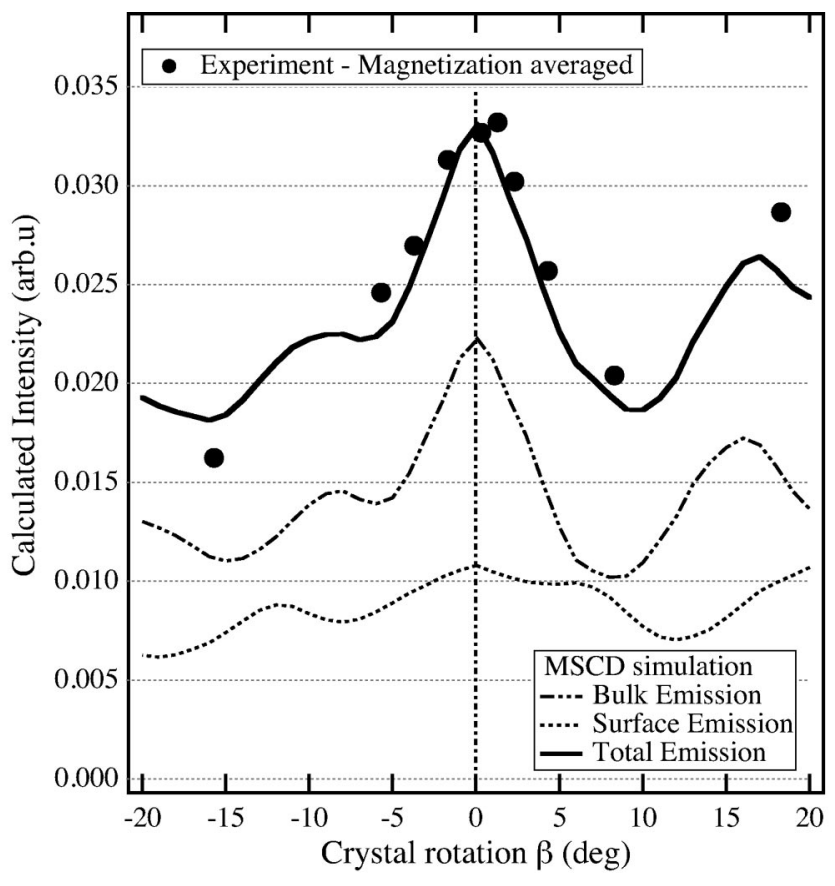

FIG. 2. Multiple-scattering calculation of the angular dependence of the relative weight between surface (dashed line) and bulk (dot-dashed) Fe $3 p$ emission from the bcc Fe(001) surface, at $h \nu$ $=165 \mathrm{eV}$. Black points are experimental data from magnetization averaged spectra. The value $\beta=0$ indicates normal emission.

(nondichroic) total PE intensity from $\mathrm{Fe}(100)$ along with our PED calculations for bcc $\mathrm{Fe}(100)$ at $h \nu=165 \mathrm{eV}$ as a function of the angle $\beta$ about the surface normal, along the [010] direction. The layer-resolved calculations show the different PED effects on the bulk and the surface Fe $3 p$ signals: the former presents an intense peak centered around normal emission, while the latter oscillates smoothly, without prominent peaks for $\beta$ values within $\pm 20^{\circ}$. The total PE intensity (magnetization averaged) data agree well with the calculated overall PED (thick line). The large difference in the calculated PED for surface and bulk as a function of angle indicates that the experimental spectra at normal emission have a much enhanced bulk contribution with respect to the data at off-normal emission. Therefore, the large modulations in the MLDAD line shape for $\mathrm{Fe} 3 p$ in this angular and energy range can be understood as relative intensity changes of bulk and surface contributions.

Figure 3 displays in three panels the experimental magnetization-averaged photoemission data sets for $\mathrm{Fe} 3 p$ in $\mathrm{Fe}(001)$ clean single crystal, Fe covered with $\sim 2 \mathrm{ML}$ of pseudomorphic $\mathrm{Co}$, and $1 \mathrm{ML}$ Fe on top of a thicker Co layer $(\sim 6 \mathrm{ML})$. The magnetic asymmetry is also shown with contour plots both on the data surfaces and on the bottom of the figure. Several factors contribute to the measured angular distribution of PE intensity, such as the differential atomic cross section, the thickness of the emitting layer, the PED effects, and the angular dependent instrumental factors due to the geometry of the experiment. As a consequence, the angular distribution of both intensity and magnetic signal is affected by the choice of the normalization procedure. Such procedure first consisted in subtracting a Shirley-like background from the raw PE data: ${ }^{23}$ the background subtracted spectrum will be referred as $I_{b s}(\theta, K E)$. Subsequently, each spectrum has been divided by the constant PE background, thus producing a real normalization for all geometrical and instrumental factors, i.e., $\quad I_{b s}(\theta, K E) / I_{\text {backg }}(\theta)$ $=I_{N o r m}(\theta, K E)$. This procedure does not alter PED modulations, since $I_{\text {backgr }}(\theta)$ was verified to be free from residual

\section{$\mathrm{Fe}(100)$}

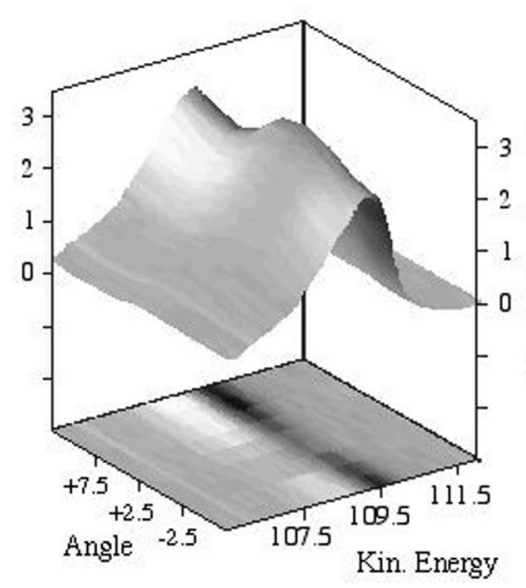

$\mathrm{Co} / \mathrm{Fe}(100)$

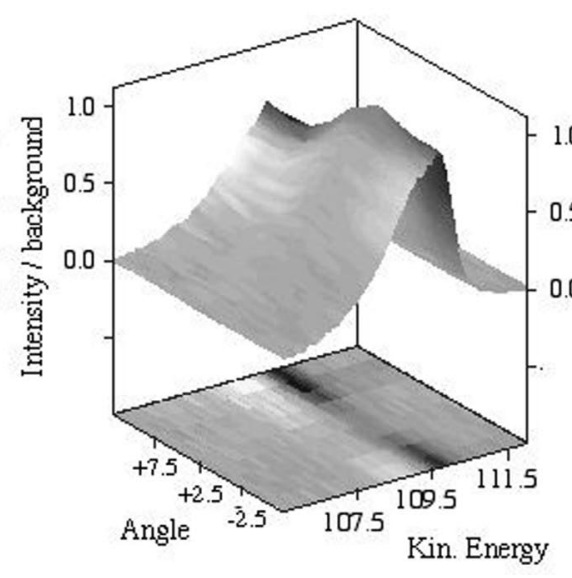

$\mathrm{Fe} / \mathrm{Co} / \mathrm{Fe}(100)$

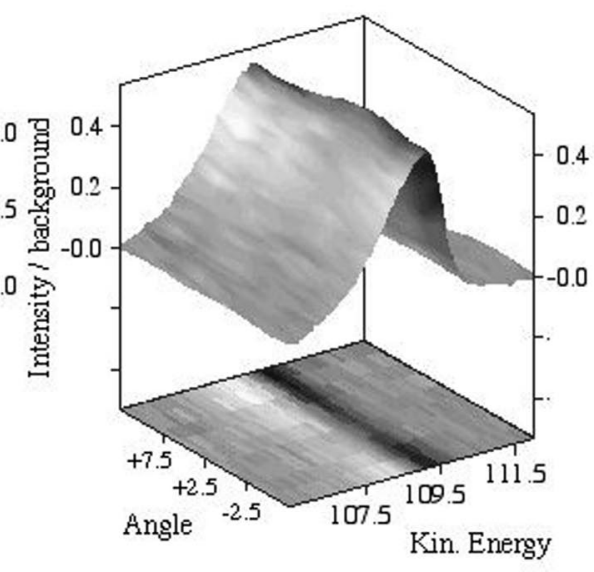

FIG. 3. Polar plots of the Fe $3 p$ PE intensities and MLDAD signals $A_{M L D A D}$ vs angle for $\mathrm{Fe}(001), \mathrm{Co} / \mathrm{Fe}(001)$, and $\mathrm{Fe} / \mathrm{Co} / \mathrm{Fe}(001)$, respectively left, center, and right panel, measured at $h \nu=165 \mathrm{eV}$. Spectra are background subtracted and divided by the background level (i.e., normalized for geometrical effects, but not for PED modulations, see text). $A_{M L D A D}$ (contour plots reported on the bottom of the figure) are differences of spectra (treated as above) and divided by the $M$-averaged integral of line shape. The angular dependence of the $A_{M L D A D}$ in $\mathrm{Fe} / \mathrm{Co} / \mathrm{Fe}(001)$, where the bulk Fe signal is almost completely removed, is much more even as well as it is the corresponding photoemission intensity. The black (white) stripes of the $A_{M L D A D}$ correspond to the maximum (minimum) signal of the dichroism. Note that for Co/Fe the $A_{M L D A D}$ reduction around normal emission is enhanced with respect to $\mathrm{Fe}(100)$. 


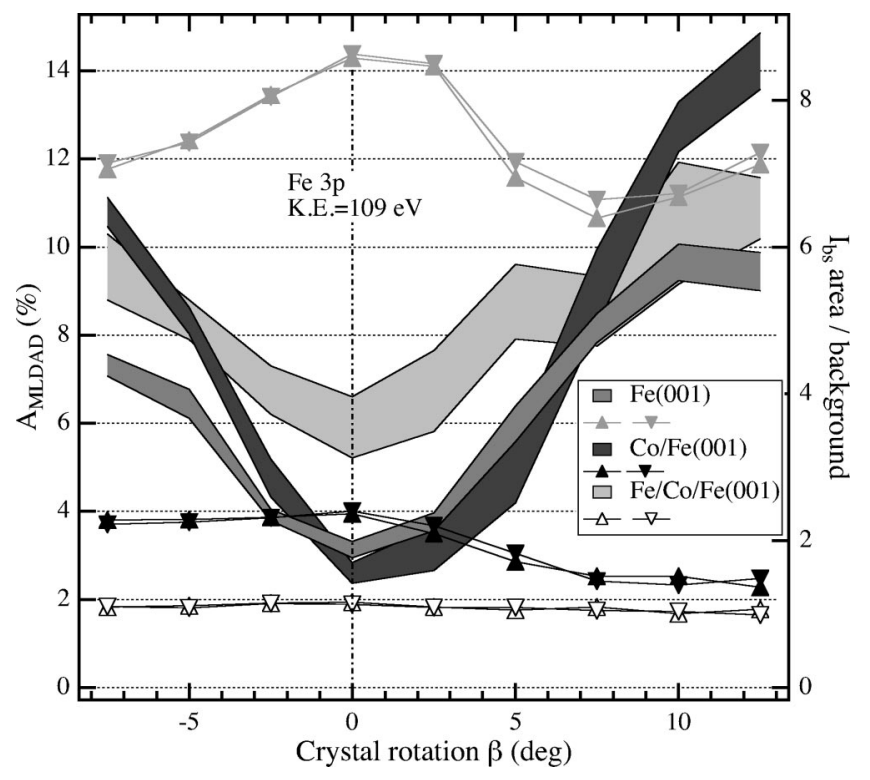

FIG. 4. Fe $3 p A_{M L D A D}$ reduction vs angle for $\mathrm{Fe}(001), \mathrm{Co} /$ $\mathrm{Fe}(001)$, and $\mathrm{Fe} / \mathrm{Co} / \mathrm{Fe}(001)$, respectively, black, dark gray, and light gray shaded areas, measured at $h \nu=165 \mathrm{eV}$. The width of the areas corresponds to the error bars. $y$ axis is the dichroic normalized intensity in percent as defined in the text. The value $\beta=0$ indicates normal emission. Up and down triangles correspond to PE intensities for the two magnetization directions.

anisotropy. The thickness of the emitting layer also modifies the angular dependence of PE, but it consists mostly of thickness dependent peak-to-background ratio, placed in a relatively small angular range around the surface normal; this can be appreciated by looking at the amplitude of background divided spectra $I_{\text {Norm }}(\theta, K E)$ shown in Fig. 3. As far as the atomic cross section is concerned, it does not provide any variation of the intensity vs angle, since the angular scans were performed at a fixed chirality. A more detailed inspection of the differences in MLDAD signals is presented in Fig. 4, where the Fe $3 p$ magnetic asymmetry $A_{M L D A D}$ (shaded areas) together with their relative total PE intensities (triangles) are reported vs angle $\beta$. For the sake of comparison, the $A_{M L D A D}$ has been defined as the amplitude of the up down spectra obtained as the normalized difference of the magnetization dependent $I_{b s}(\theta)$ spectra, i.e., normalized to the sum of the up-down peak area,

$$
\begin{gathered}
\operatorname{MLDAD}(\theta, K E)=\frac{I_{b s}(\theta, K E)^{\mathrm{up}}-I_{b s}(\theta, K E)^{\mathrm{down}}}{\operatorname{area}(\theta)^{\mathrm{up}}+\operatorname{area}(\theta)^{\mathrm{down}}} \\
A_{M L D A D}(\theta)=\max [\operatorname{MLDAD}(\theta)]-\min (\operatorname{MLDAD}(\theta)] .
\end{gathered}
$$

This produces an effective magnetization independent averaging of the intensity. The advantage of such definition lies in the fact that $A_{M L D A D}$ are normalized for all the angular dependences of PE, making it possible to compare results obtained on the three different systems. This procedure also preserves the noise level of the difference spectra, which would be highly enhanced if raw asymmetry is used.
In the case of clean $\mathrm{Fe}(001)$ crystal the decrease of the MLDAD signal concurs with the enhancement of the total intensity at $\beta=0$. The $\mathrm{Co} / \mathrm{Fe}(001)$ sample shows the same effect, but more pronounced because of the removal of the surface contribution to the $\mathrm{Fe}$ signal. Conversely, the angular dependence of the total photoemission intensity for the $\mathrm{Fe} /$ $\mathrm{Co} / \mathrm{Fe}(001)$ sample, where the bulk Fe signal is almost completely removed, is much more evenly distributed.

The magnetic signal measured along the surface normal undergoes a reduction in all the three cases: the dip is the largest for $\mathrm{Fe} 3 p$ MLDAD in $\mathrm{Co} / \mathrm{Fe}(100)$, and it is the weakest in $\mathrm{Fe} / \mathrm{Co} / \mathrm{Fe}$. The link between PED modulation of the bulk intensity, as calculated in Fig. 2, and the variation of the dichroic signal is clear. From the $\mathrm{Co} / \mathrm{Fe}$ and $\mathrm{Fe} / \mathrm{Co} / \mathrm{Fe} \mathrm{Fe} 3 p$ data one can attempt to estimate the bulk sensitivity obtained in our experiment. In fact, a minimum in MLDAD at normal emission is found also for the top $\mathrm{Fe}$ layer of $\mathrm{Fe} / \mathrm{Co} / \mathrm{Fe}$ (Fig. 4). The presence of this minimum, although less pronounced with respect to the other two systems, sets our actual level of sensitivity in separating the bulk from the surface contribution. The decrease between the off-normal and normal emission MLDAD values is $82.5 \pm 2.5 \%, 67.5 \pm 3.5 \%$, and 41 $\pm 5 \%$, respectively, for $\mathrm{Co} / \mathrm{Fe}, \mathrm{Fe}(100)$ clean and $\mathrm{Fe} / \mathrm{Co} / \mathrm{Fe}$. The $A_{M L D A D}$ values obtained for $\mathrm{Co} / \mathrm{Fe}$ and $\mathrm{Fe} / \mathrm{Co} / \mathrm{Fe}$ reproduce well the ones of the clean $\mathrm{Fe}(100)$ within the error bars, taking into account the escape depth of photoelectrons in the range of kinetic energies $(\sim 5 \AA)$. Figure 5 presents a lineshape analysis of $\mathrm{Fe} 3 p$ PE data taken with opposite magnetization (mirror spectra) for the three samples. By assuming that the normal emission spectra are mostly representative of the bulk contribution, due to the strong PED enhancement, a line-shape reduction can be done by calibrating the amount of forward-scattered signal in each of the experiments. By means of such a procedure one filters out the bulk contribution from the total, leaving out the surface line-shape contribution. As the normal emission data also contain some surface contribution this analysis is not directly useful to assess the intensity of the surface peak. Nevertheless the accuracy of the line-shape analysis is only limited by the data quality in terms of energy resolution and statistics. The bulklike intensities have been filtered out by subtracting the magnetization dependent spectra measured at normal emission from the corresponding off-normal ones (measured at $7.5^{\circ}$ off normal), for $\mathrm{Fe}(100), \mathrm{Co} / \mathrm{Fe}(100)$, and $\mathrm{Fe} / \mathrm{Co} / \mathrm{Fe}(100)$. The resulting line shapes have a striking resemblance with magnetization dependent Fe $3 p$ core level measured off normal and their energy positions are identical, within the error bar, for all the three systems, confirming the hypothesis of a bulklike contribution. The presence of the small residual intensity for the $\mathrm{Fe} / \mathrm{Co} / \mathrm{Fe}$, which is responsible for the dip at normal emission, may be due to either a small contribution from the underneath $\mathrm{Fe}(100)$ substrate or an island growth with different island thicknesses onto the Co.

The same analysis for Co $3 p$ PE intensity and its relative MLDAD signal is presented in Fig. 6 for $h \nu=165 \mathrm{eV}$ and $h \nu=170 \mathrm{eV}$. In the latter case, Co $3 p$ photoelectrons do have the same kinetic energy as $\mathrm{Fe} 3 p$ measured at $h \nu$ $=165 \mathrm{eV}$. The results confirm the overall behavior observed for $\mathrm{Fe} 3 p$. The minimum in the angular scan is found to be 

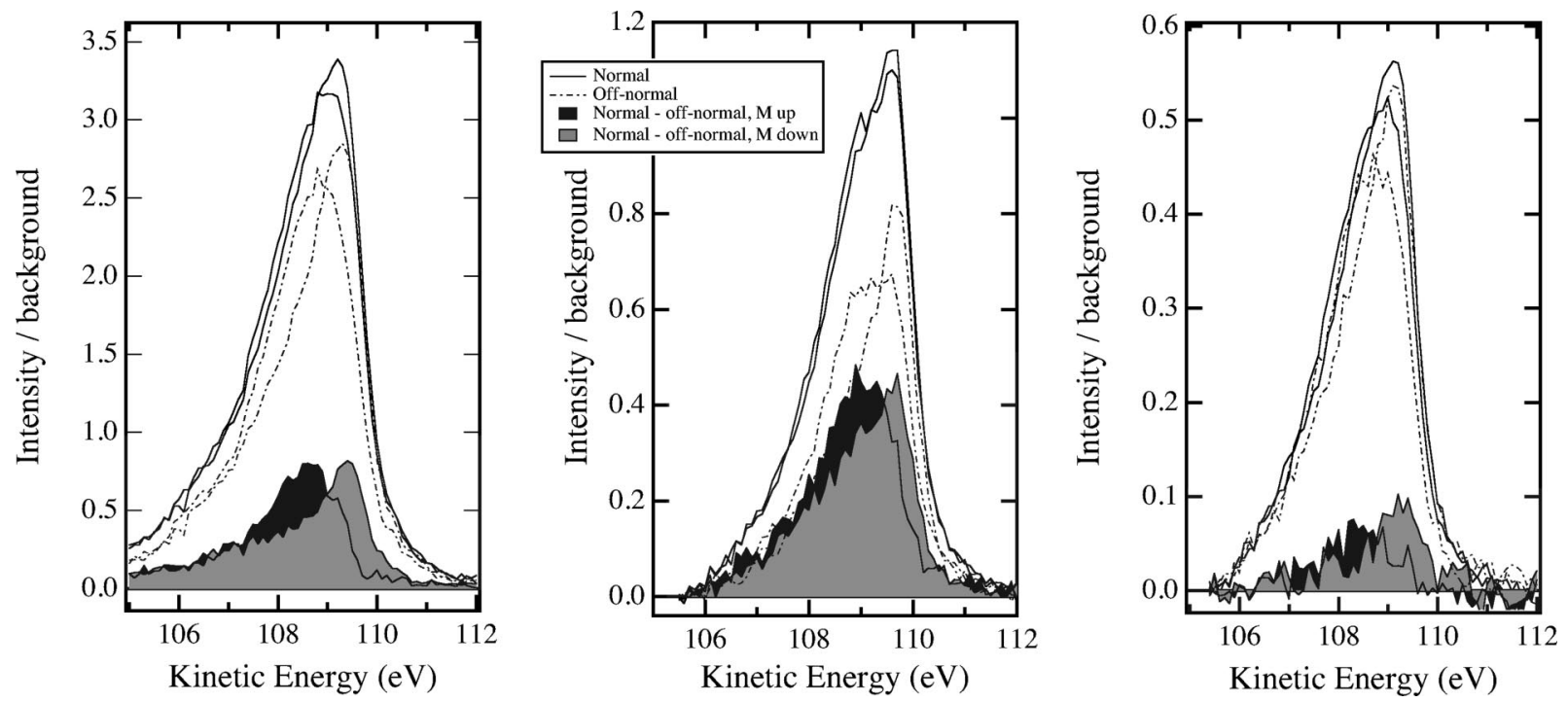

FIG. 5. Filtered intensities (filled areas) obtained by subtracting normal emission (lines) from off normal by $7.5^{\circ}$ (dash dot) Fe $3 p$ MLDAD spectra. From left to right, $\mathrm{Fe}(100), \mathrm{Co} / \mathrm{Fe}(100), 1 \mathrm{ML} \mathrm{Fe} / 6 \mathrm{ML} \mathrm{Co} / \mathrm{Fe}(100)$. All spectra are normalized as defined in text. The subtracted intensities correspond to the forward-scattering contribution responsible for the MLDAD decrease at normal emission.

more pronounced for the thick layer underneath the top $\mathrm{Fe}$ film, and less pronounced for the two monolayers grown on $\mathrm{Fe}(100)$. Noteworthly in both Co $3 p$ and Fe $3 p$ cases the minimum of the MLDAD signal occurs at normal emission $(\beta=0)$, if the photoelectron kinetic energies are identical. This is not the case for Co $3 p$ MLDAD measured at $h \nu$ $=165 \mathrm{eV}$, where the minimum of MLDAD is found at $\beta$ $=2^{\circ}$. These facts further confirm the large PED influence on MLDAD results in the energy range most relevant for surface sensitive studies. ${ }^{22}$ PED effects in MLDAD are found in an extended kinetic-energy range, ${ }^{22}$ from 60 to $200 \mathrm{eV}$. The MLDAD oscillations depend on both kinetic energy and angle: the dip in angular dependence is shifted away from normal emission if the kinetic energy is changed, as confirmed both for $\mathrm{Co}$ and $\mathrm{Fe}$ and $\mathrm{Ni}^{32}$ Accordingly, for experiments performed away from this particular zone of kinetic energy, one expects that the MLDAD signal should follow the predicted atomic energy dependence and that the dip of MLDAD vs angle should be absent at normal emission even for crystalline materials. This is confirmed in Fig. 7, where the Fe $3 p A_{M L D A D}$ vs angle is presented for the $\mathrm{Fe} / \mathrm{Co} / \mathrm{Fe}$ at $h \nu=220 \mathrm{eV}$. Within the error bars, no oscillation is found in the magnetic signal, as well as in the total PE intensity (open triangles). This confirms our interpretation of the relationship between bulk and surface contributions in MLDAD-PED: the MLDAD geometry must be carefully chosen as a function of the used kinetic energy range, in order to avoid misleading interpretation of the magnetic signals. Same results have been found at this photon energy for Co $3 p$ and $\mathrm{Fe} 3 p$ in both $\mathrm{Co} / \mathrm{Fe}$ and clean $\mathrm{Fe}(100)$. Full multiple-scattering calculations reveal that at $h \nu=220 \mathrm{eV}$ (a) the enhancement of the bulk peak in the PE intensity is found at angles $\beta=+10 /+15$; (b) the bulk intensity is placed asymmetrically with respect to normal emission, due to the geometry of present experiment. It is worth noticing that this geometry corresponds to the maximum MLDAD signal in terms of chirality.
It should be noted that a difference of the present experimental results with respect to those previously reported concerns the Fe $3 p$ MLDAD data from $\operatorname{Fe}(100)$ at $\beta=0$. A clear sign reversal in the MLDAD was observed around $\beta$ $=0$ in Refs. 22 and 25, at $h \nu=165 \mathrm{eV}$ and $h \nu=1484 \mathrm{eV}$, respectively. While in our case only a severe reduction of the MLDAD but no sign reversal is found. The main differences in the experimental conditions are the angular resolution of the energy analyzers employed and the sample temperature that was $300 \mathrm{~K}$ in the present experiment and $100 \mathrm{~K}$ when the sign reversal was reproducibly observed [both in $\mathrm{Fe}(100)$ and in Fe-Ni alloys on $\mathrm{Fe}(100)]$. The influence of temperature, i.e., Debye-Waller factor, has been already put forward as the reason for this difference, ${ }^{25}$ being the loss in scattering intensity expected to be smaller at $100 \mathrm{~K}$ than at room temperature.

\section{CONCLUSIONS}

From the analysis of the angular and energy dependence of the Fe $3 p$ MLDAD in three different crystalline environment we were able to identify the relationship between PED effects and MLDAD variations. PED clearly influences magnetic dichroism data in photoemission experiments. The intensity changes can be understood with the kinematic model analysis of PED when the photoelectron energies exceed several hundred $\mathrm{eV}$. But in surface sensitive experiments the lower values of kinetic energies impose to analyze PED by means of full multiple-scattering calculations. Such calculations have been performed and presented in a layer selective mode. It turns out that for an angular range of $\pm 20^{\circ}$ about normal emission and kinetic energies of about $100 \mathrm{eV}$ the PED effects on photoelectrons originating from the surface are weak while the PED effects on the bulk signal dominate. In particular, at normal emission, around $h \nu=165 \mathrm{eV}$, the bulk peak dominates over the surface peak; comparable intensities are calculated off normal. Moreover, the strong de- 

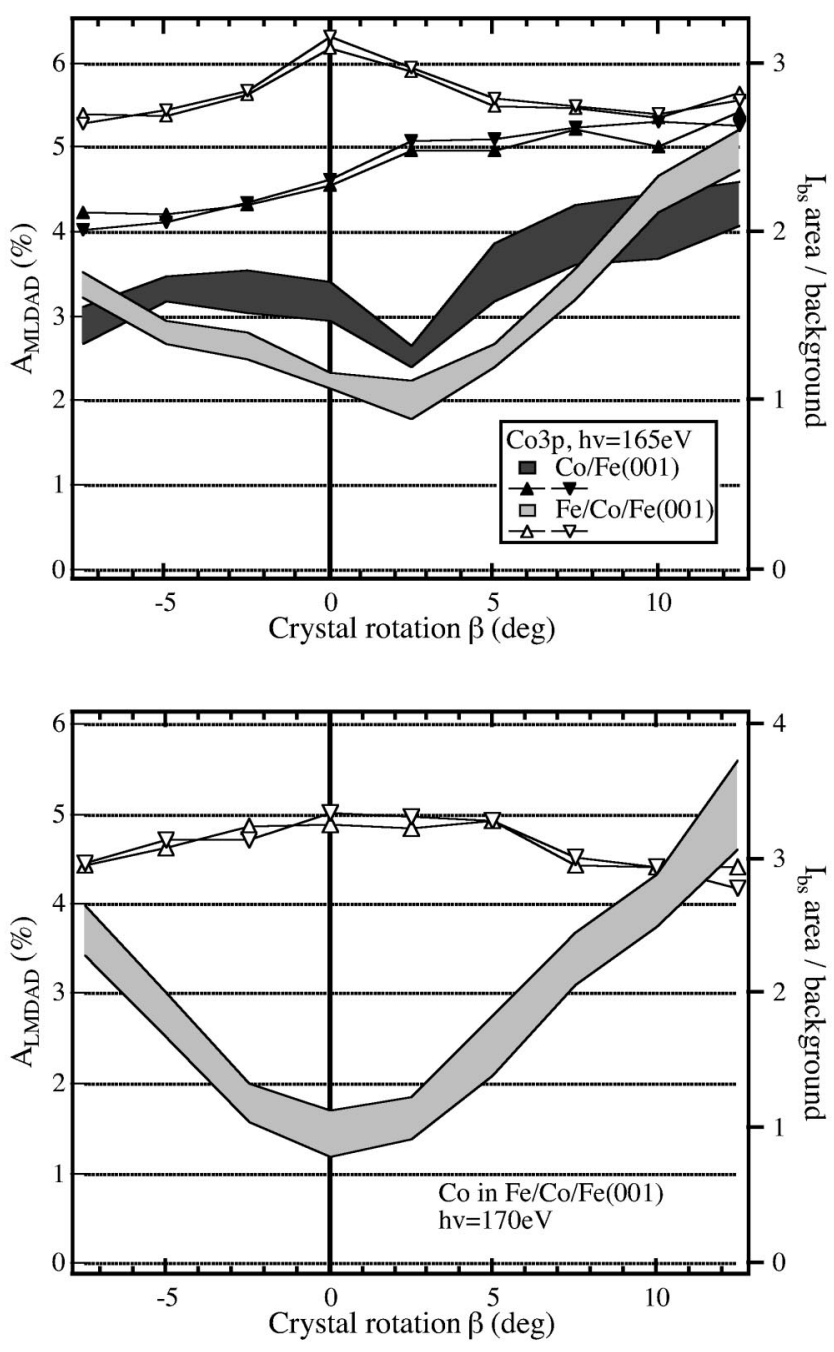

FIG. 6. Co $3 p A_{M L D A D}$ reduction vs angle for $\mathrm{Co} / \mathrm{Fe}(001)$ and $\mathrm{Fe} / \mathrm{Co} / \mathrm{Fe}(001)$, respectively, dark gray, and light gray shaded areas, measured at $h \nu=165 \mathrm{eV}$ (top panel) and $h \nu=170 \mathrm{eV}$ (bottom panel). The width of the areas corresponds to the error bars. The $y$ axis is the dichroic normalized intensity in percent as defined in the text. The value $\beta=0$ indicates normal emission. Up and down triangles correspond to PE intensities for the two magnetization directions. The minimum of the Co $3 p A_{M L D A D}$ is found at normal emission only for $h \nu=170 \mathrm{eV}$, i.e., only when Co $3 p$ are at the same kinetic energy of Fe $3 p$ measured at $h \nu=165 \mathrm{eV}$.

crease of MLDAD signal is found at normal emission only at specific values of both emission angle and kinetic energy. The line-shape changes are therefore due to the surface corelevel shift, to the different PE and MLDAD line shape of the

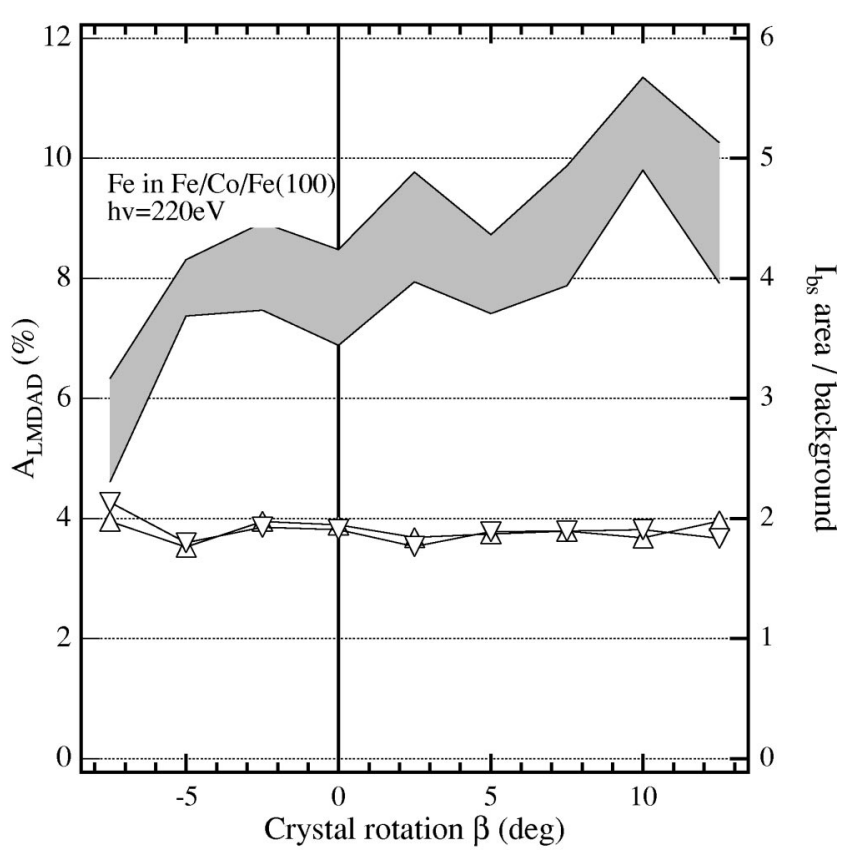

FIG. 7. Fe $3 p A_{M L D A D}$ vs angle for $\mathrm{Fe} / \mathrm{Co} / \mathrm{Fe}(001)$ (light gray shaded area), and its relative PE intensity vs angle (open triangles) measured at $h \nu=220 \mathrm{eV}$. The width of the area corresponds to the error bars. $y$ axis is the dichroic normalized intensity in percent as defined in the text. The value $\beta=0$ indicates normal emission. No oscillation or dip is found around normal emission for both $A_{M L D A D}$ and total PE.

two components, to the different magnetic splitting of bulk and surface, and to the different phase shift in each MLDAD spectrum. The large PED oscillations around normal emission disappear on increasing the photon energy. The overall measured line shape of TM core level is therefore the sum of two different line shapes, energy shifted, and with different relative intensities due to PED.

Our present results, while not resolving all of the relevant aspects of the surface magnetic response, provide a clear evidence that surface and bulk photoemission magnetic dichroism contribute significantly to the measured data. Thanks to the action of PED, we have shown that the line shape of the bulk contribution can be retrieved from energy and angle-dependent chiral experiment. The possibility of extracting filtered line shapes for bulk and surface atoms allows one to apply a rationale for establishing the relative changes of the orbital magnetic moment, and possibly the relative changes of the energy splitting, connected to the spin magnetic moments.
${ }^{1}$ H. C. Siegmann, J. Phys.: Condens. Matter 4, 8935 (1992).

${ }^{2}$ J. C. Walker et al., J. Appl. Phys. 55, 2500 (1984).

3 J. Korecki and U. Gradmann, Hyperfine Interact. 28, 931 (1986).

${ }^{4}$ C. S. Wang and A. J. Freeman, Phys. Rev. B 24, 4364 (1981).

${ }^{5}$ C. L. Fu, A. J. Freeman, and T. Oguchi, Phys. Rev. Lett. 54, 2700 (1985).
${ }^{6}$ O. Eriksson et al., Solid State Commun. 78, 801 (1991).

${ }^{7}$ C. S. Fadley et al., Prog. Surf. Sci. 54, 341 (1997), and references therein.

${ }^{8}$ Ch. Roth, F. U. Hillebrecht, H. Rose, and E. Kisker, Phys. Rev. Lett. 70, 3479 (1993); Ch. Roth, H. Rose, F. U. Hillebrecht, and E. Kisker, Solid State Commun. 86, 647 (1993). 
${ }^{9}$ F. Sirotti, G. Panaccione, and G. Rossi, Phys. Rev. B 52, R17 063 (1995)

${ }^{10}$ H. B. Rose et al., in Spin-Orbit Influenced Spectroscopies of Magnetic Solids, edited by H. Ebert and G. Scuhtz, Lecture Notes in Physics Vol. 466 (Springer-Verlag, Berlin, 1996), p. 105.

${ }^{11}$ G. van der Laan and B. T. Thole, Phys. Rev. B 48, 210 (1993).

${ }^{12}$ G. van der Laan, Phys. Rev. B 51, 240 (1995).

${ }^{13}$ G. van der Laan, Phys. Rev. B 55, 3656 (1997).

${ }^{14}$ D. Venus, Phys. Rev. B 49, 8821 (1994).

${ }^{15}$ N. A. Cerepkov, Phys. Rev. B 50, 13813 (1994).

${ }^{16}$ K. Starke et al., in Spin-Orbit Influenced Spectroscopies of Magnetic Solids (Ref. 10), p. 65.

${ }^{17}$ G. Panaccione, P. Torelli, G. Rossi, G. van der Laan, M. Sacchi, and F. Sirotti, Phys. Rev. B 58, R5916 (1998).

${ }^{18}$ G. Rossi, F. Sirotti, N. A. Cherepkov, F. Combet-Farnoux, and G. Panaccione, Solid State Commun. 90, 557 (1994).

${ }^{19}$ G. Rossi, G. Panaccione, and F. Sirotti, Phys. Rev. B 55, 11488 (1997).

${ }^{20}$ F. U. Hillebrecht, H. B. Rose, T. Kinoshita, Y. U. Idzerda, G. van der Laan, R. Denecke, and L. Ley, Phys. Rev. Lett. 75, 2883 (1995).

${ }^{21}$ R. Schellenberg, E. Kisker, A. Fanelsa, F. U. Hillebrecht, J. G. Menchero, A. P. Kaduwela, C. S. Fadley, and M. A. Van Hove, Phys. Rev. B 57, 14310 (1998).
${ }^{22}$ G. Panaccione, F. Sirotti, and G. Rossi, Solid State Commun. 113, 373 (2000).

${ }^{23}$ D. A. Shirley, Phys. Rev. B 5, 4709 (1972).

${ }^{24}$ F. U. Hillebrecht, Ch. Roth, H. B. Rose, W. G. Park, E. Kisker, and N. A. Cherepkov, Phys. Rev. B 54, 15618 (1996).

${ }^{25}$ X. Gao, M. Salvietti, W. Kuch, C. M. Schneider, and J. Kirschner, J. Electron Spectrosc. Relat. Phenom. 113, 137 (2001).

${ }^{26}$ L. Floreano, G. Naletto, D. Cvetko, R. Gotter, M. Malvezzi, L. Marassi, A. Morgante, A. Santaniello, A. Verdini, F. Tommasini, and G. Tondello, Rev. Sci. Instrum. 70, 3855 (1999); see also the website www.tasc.infm.it/tasc/lds/aloisa.html

${ }^{27}$ J. Wider, F. Baumberger, M. Sambi, R. Gotter, A. Verdini, F. Bruno, D. Cvetko, A. Morgante, T. Greber, and J. Osterwalder, Phys. Rev. Lett. 86, 2337 (2001).

${ }^{28}$ F. Bruno, D. Cvetko, L. Floreano, R. Gotter, A. Morgante, A. Verdini, G. Panaccione, M. Sacchi, P. Torelli, and G. Rossi, J. Magn. Magn. Mater. 233, 123 (2001).

${ }^{29}$ This code is freely distributed at http electron.lbl.gov/mscdpack/ mscdpack.html

${ }^{30}$ F. Bruno, D. Cvetko, L. Floreano, R. Gotter, C. Mannori, L. Mattera, R. Moroni, S. Prandi, S. Terreni, A. Verdini, and M. Canepa, Appl. Surf. Sci. 162, 340 (2000).

${ }^{31}$ S. K. Kim, C. Petersen, F. Jona, and P. M. Marcus, Phys. Rev. B 54, 2184 (1996).

${ }^{32}$ F. Sirotti and P. Torelli (unpublished). 